\title{
Advances in the Parameter Space Concept for Crystal Structure Determination - a maximum resolution study
}

\author{
Matthias Zschornak $^{1,2}$, Christian Wagner ${ }^{1,2}$, Melanie Nentwich $^{3}$, Dirk C. Meyer ${ }^{1}$, Karl Fischer $^{4}$ \\ ${ }^{1}$ TU Bergakademie Freiberg, Institut für Experimentelle Physik, Leipziger Str. 23, D-09599 Freiberg, Germany, \\ ${ }^{2}$ TU Chemnitz, Institut für Physik, Reichenhainer Str. 70, D-09126 Chemnitz, Germany, \\ ${ }^{3}$ DESY Photon Science, Notkestr. 85, D-22607 Hamburg, Germany, \\ ${ }^{4}$ Universität des Saarlandes, Experimentalphysik, Campus A5 1, D-66041 Saarbrücken, Germany
}

matthias.zschornak@tu-freiberg.de

Within the last 15 years, the Parameter Space Concept (PSC) was theoretically developed by Fischer, Kirfel and Zimmermann as an alternative approach to solve crystal structures from diffraction intensities without use of Fourier transforms [1-6]. Each experimentally determined reflection restricts the $3 N$-dim. parameter space of atomic coordinates for a crystal structure solution $(N$ atoms $)$ by a manifold of $3 \mathrm{~N}-1$ dimensions, equivalent to a unique isosurface, whereas the true solution vector will be the intersection of all isosurfaces. The method has already been tested on numerous, partly challenging problems of X-ray diffraction.

We present a study of the maximum resolution of the PSC. As an example, a split position of La and $\mathrm{Sr}$ with $(0,0, z=0.3584)$ has been investigated in the potential high-temperature super-conductor $\left(\mathrm{La}_{0.5} \mathrm{Sr}_{1.5}\right) \mathrm{MnO}_{4}, \mathrm{I} 4 / \mathrm{mmm}$. A positional shift of the cations in the order of $\Delta z \approx 0.001_{5}(\approx 0.02 \AA)$ has been suggested in literature [7]. Enhancing the scattering difference of La and $\mathrm{Sr}$ by $f^{\prime}$ Sr, this split was later verified using the PSC within a rather conservative model test [8]. As a result a shift $\Delta z=0.01_{3}$ had been determined. We now add to the discussion an evaluation based on two additional model data sets, each with $(00 l)$ reflections $(l=2,4 \ldots 20)$ and varied relative errors of up to $20 \%$. A graphical representation of the parameter space revealed an improvement of resolution with a shift of $\Delta z=0.01_{2} \ldots 0.01_{6}$ $\left(\approx 0.15 \ldots 0.2_{0} \AA\right)$. Due to the difference in scattering power of $\mathrm{La}$ and $\mathrm{Sr}$, a pseudosymmetric structure solution $\left(z_{\mathrm{La}}^{*}, z_{\mathrm{Sr}}^{*}\right)$ exists for approximately interchanged $z$-positions, which we discuss in conjunction with the accurate solution $\left(z_{\mathrm{La}}, z_{\mathrm{Sr}}\right)$. The two solutions were defined by the intersection of isolines representing $(00 l)$ reflection intensities [9]. There is a non-vanishing variance of the pseudosymmetric structure solution, whereas the accurate solution does not vary. Depending on the relative error of the diffraction intensities, we present respective resolution limits for the split position.
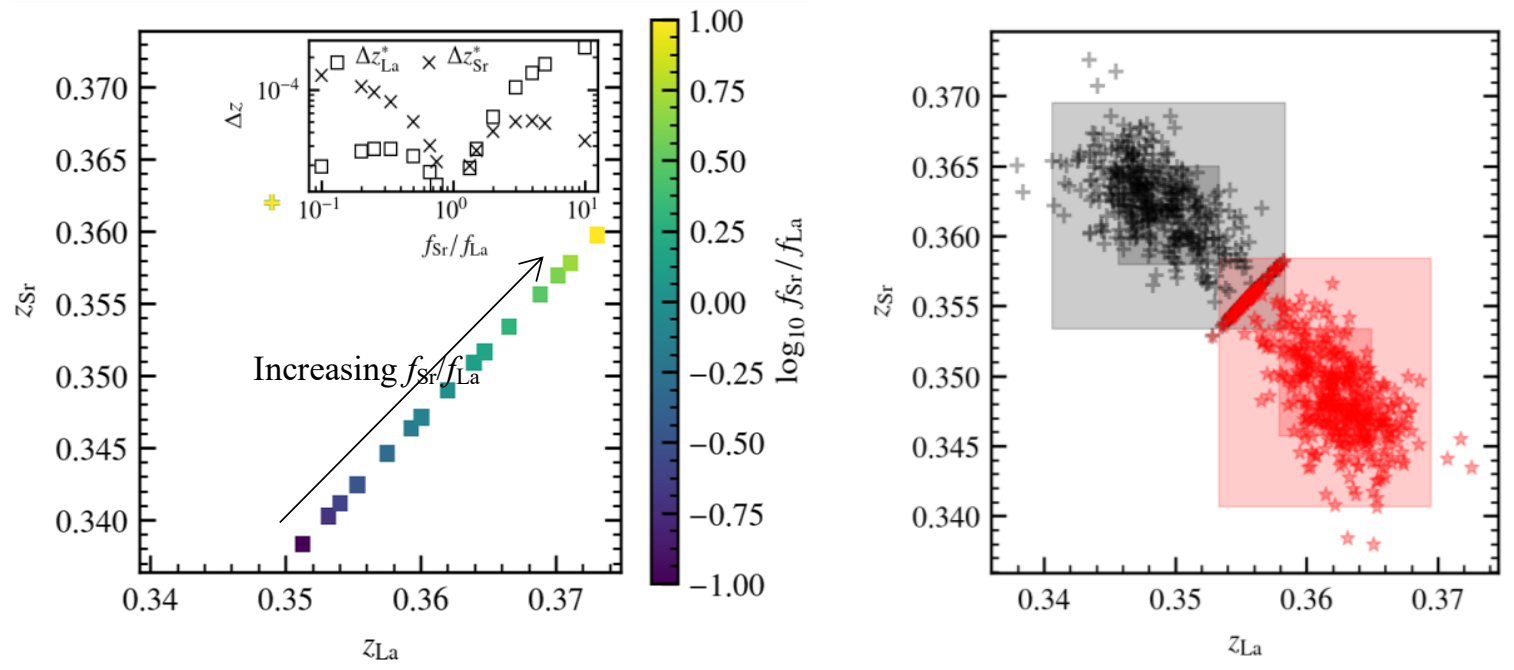

Figure 1. Left: Model study of the real $(0.362 \mid 0.349)$ and the (broken) pseudosymmetric structure solution $\left(z_{\mathrm{La}}^{*}, z_{\mathrm{Sr}}^{*}\right)$ of the PSC model as a function of the relative scattering strength of the La and $\mathrm{Sr}$ atom. Inset: Variance of the (broken) pseudosymmetric structure solution $\Delta z_{\mathrm{La}, \mathrm{Sr}}^{*}$ as a function of the relative scattering strength of the $\mathrm{La}$ and $\mathrm{Sr}$ atom. The real solution shows no variance. Right: Monte-CarloStudy of the structure solution as a function including the single (2.3-times) trust region for $20 \%$ intensity error and equal scatterers. 
[1,2] K.F. Fischer, A. Kirfel, H. Zimmermann, (2005) Z. Krist. 220, 643; (2008) Croatica Chemica Acta 81, 381.

[3,4] A. Kirfel, K.F. Fischer, H. Zimmermann, (2006) Z. Krist. 221, 673; H. Zimmermann, K.F. Fischer, (2009) Acta Cryst. A65, 443.

[5,6] A. Kirfel, K. F. Fischer, (2009) Z. Krist. 224, 325; (2010) Z. Krist. 225, 261.

[7,8] T. Lippmann et al., (2003) HASYLAB Jahresberichte 583; A. Kirfel, K. F. Fischer, (2004) Z. Krist. Suppl. $21,101$.

[9] M. Zschornak, M. Nentwich, D.C. Meyer, A. Kirfel, K. Fischer, (2020) $27^{\text {th }}$ Annual Meeting of the DGK, Wrocław, Poland.

Keywords: High resolution, high quality, validation and reproducibility in structural science, $\mathrm{X}$-ray diffraction

M.Z., C.W., D.M., and K.F. acknowledge funding by the DFG within the project DFG 442646446, ZS 120/5-1. 